\title{
A CLASS OF MAXIMAL ORDERS INTEGRAL OVER THEIR CENTRES
}

\author{
by ANDY J. GRAY
}

(Received 9 March, 1982)

1. Introduction. In a recent paper [1], Brown, Hajarnavis and MacEacharn have considered non-commutative Noetherian local rings of finite global dimension which are integral over their centres. For such a ring $R$ they have shown:

(i) $R$ is a prime ring whose Krull and global dimensions coincide;

(ii) $R=\cap R_{p}$ where $p$ runs through the set of rank one primes of the centre of $R$, and each $R_{\mathrm{p}}$ is hereditary;

(iii) the centre of $R$ is a Krull domain.

We shall show that each $R_{\mathrm{p}}$ in (ii) above is in fact a principal right and left ideal ring. We deduce that the above ring $R$ is a maximal order (defined below), and that if $R$ is in addition a PI-ring with centre $Z$ then it is a maximal $Z$-order in the sense of Fossum [5].

Our result covers the case where $R$ is a local Noetherian ring of finite global dimension finitely generated as a module over its centre, which has previously been discussed in [7], and indeed our proof is somewhat easier than that given there. However, let $D$ be a division ring which is locally finite dimensional, but not finite dimensional, over its centre. Then the localization of the polynomial ring $D\left[X_{1}, \ldots, X_{n}\right]$ at the maximal ideal generated by $X_{1}, \ldots, X_{n}$ is a local Noetherian ring of global dimension $n$ which is integral, but not finitely generated, over its centre. The reader will find further details in $[\mathbf{1}, 7.1]$.

Throughout, all rings will be assumed to have an identity, and Noetherian will mean left and right Noetherian. A ring $R$ with Jacobson radical $J$ is called semilocal (respectively local) if $R / J$ is semisimple (respectively simple) Artinian. For a right $R$-module $M$, $M^{\oplus s}$ denotes a direct sum of $s$ copies of $M$.

I should like to thank my supervisor Dr. C. R. Hajarnavis for his help and encouragement, and Dr. A. W. Chatters for many useful conversations.

2. The Main Theorem. We shall first briefly define maximal orders (in the sense of Asano). Details may be found in [6].

Let $R$ be a ring with a simple Artinian (right and left) quotient ring $Q$. A subset $I$ of $Q$ is called a right $R$-ideal if:

(i) $I$ is a right $R$-submodule of $Q$;

(ii) $I$ contains a unit of $Q$;

(iii) $u I \subset R$ for some unit $u$ of $Q$.

Left $R$-ideals and (two-sided) $R$-ideals are defined in the obvious fashion. Clearly any non-zero ideal of $R$ is an $R$-ideal. If $I$ is an $R$-ideal, write

$$
\begin{aligned}
& O_{r}(I)=\{q \in Q \mid I q \subset I\} \\
& O_{l}(I)=\{q \in Q \mid q I \subset I\} .
\end{aligned}
$$

Glasgow Math. J. 24 (1983) 177-180. 
Then $R$ is called a maximal order if $O_{r}(I)=R=O_{t}(I)$ for each $R$-ideal $I$ of $Q$. We note by $[6,3.1], R$ is a maximal order precisely when $O_{r}(I)=R=O_{l}(I)$ for each non-zero (ordinary) ideal $I$ of $R$. 10.2].

We begin with the following lemma, whose proof may be found in, for example, [4,

LEMMA 1. Let $R$ be any ring, $J$ its Jacobson radical, and $P$ and $Q$ finitely generated (f.g.) projective right $R$-modules. If $P / P J$ is an $R / J$-module direct summand of $Q / Q J$, then $P$ is a direct summand of $Q$.

Since a local ring has a unique simple right module (up to isomorphism), it follows that such a ring has a unique f.g. projective indecomposable right module. We shall, however, wish to apply Lemma 1 to certain semilocal localizations of a local ring, and thus require:

LemMA 2. Let $R$ be a right Noetherian ring of finite right global dimension, and suppose that $R$ has a unique f.g. projective indecomposable right module $P$. Let $S=R_{g}$ be the classical localization of $R$ at a right Ore set $\mathscr{T}$ of regular elements. Suppose that $S$ is semilocal. Then $S$ has a unique f.g. projective indecomposable right module, namely $P \otimes_{R} S$.

Proof. Let $Q$ be a f.g. projective indecomposable right $S$-module. We can write $Q=q_{1} S+\ldots+q_{i} S$ with each $q_{i} \in Q$. Let

$$
K=q_{1} R+\ldots+q_{\mathrm{t}} R,
$$

and form an $R$-projective resolution

$$
0 \rightarrow P_{n} \rightarrow P_{n-1} \rightarrow \ldots \rightarrow P_{0} \rightarrow K \rightarrow 0 .
$$

Each $P_{i}$ can be chosen finitely generated, and hence is a direct sum of copies of $P$. Since $K \otimes_{R} S \cong Q$ and ${ }_{R} S$ is flat, we have an exact sequence of $S$-modules

$$
0 \rightarrow P_{n} \otimes_{R} S \rightarrow \ldots \rightarrow P_{0} \otimes_{R} S \rightarrow Q \rightarrow 0 .
$$

As $Q$ is $S$-projective an easy induction on the length of this resolution shows that there are integers $k$ and $l$ such that

$$
\left(P \otimes_{R} S\right)^{\oplus k} \oplus Q \cong\left(P \otimes_{R} S\right)^{\oplus l}
$$

If $J$ is the Jacobson radical of $S$, we obtain

$$
\frac{\left(P \otimes_{R} S\right)^{\oplus k}}{\left(P \bigotimes_{R} S\right)^{\oplus k} . J} \oplus \frac{Q}{Q J} \cong \frac{\left(P \otimes_{R} S\right)^{\oplus l}}{\left(P \otimes_{R} S\right)^{\oplus l} . J}
$$

Comparing the simple modules occurring, we must therefore have

$$
\frac{Q}{Q J} \cong \frac{\left(P \otimes_{R} S\right)^{\oplus(l-k)}}{\left(P \otimes_{R} S\right)^{\oplus(l-k)} \cdot J}
$$

From Lemma 1 and the indecomposability of $Q$ we deduce $Q \cong P \bigotimes_{R} S$, as required. 
We fix some notation. For the remainder of the paper, $R$ will be a local Noetherian ring of finite global dimension integral over its centre $Z$. Further, $\mathfrak{B}$ will denote the set of rank one primes of $Z$. We can now prove:

Proposition 3. For each $p \in \mathfrak{B}, R_{\mathrm{p}}$ is a principal left and right ideal ring.

Proof. By the result quoted in the introduction, $R_{p}$ is certainly a hereditary Noetherian prime ring, and is semilocal by $[3,2.2]$. Let $I$ be a non-zero right ideal of $R_{\mathrm{p}}$. We are to prove that $I$ is principal, and so we may assume that $I$ is essential as a right ideal of $R_{p}$. By Lemma $2, R_{p}$ has a unique f.g. projective indecomposable right module $Q$, and so $I \cong Q^{\oplus s}$ for some $s$. Also, $R \cong Q^{\oplus t}$ for some $t$. Since the uniform dimensions of $I_{R}$ and $R_{R}$ are equal, we have $s=t$ and $I$ is right principal.

We are in a position to obtain our main result.

THEOREM 4. $R$ is a maximal order.

Proof. We have $R=\bigcap_{p \in \mathfrak{P}} R_{\mathrm{p}}$ by $[\mathbf{1}, 6.7]$, and by Proposition 3 each $R_{\mathrm{p}}$ is a principal left and right ideal ring. If now $I$ is a non-zero ideal of $R$ and $q$ lies in the quotient ring of $R$,

$$
q I \subset I \Rightarrow q I R_{\mathrm{p}} \subset I R_{p} \quad \text { for each } \quad p \in \mathfrak{B} \Rightarrow q \in \bigcap_{p \in \mathfrak{B}} R_{p}
$$

since $I R_{\mathrm{p}}$ is an invertible ideal of $\boldsymbol{R}_{\mathrm{p}}$. Thus $\boldsymbol{R}$ is a maximal order by $[6,3.1]$.

Theorem 4 fails should the requirement that $R$ be local be weakened to one of semilocality. To see this, let $S$ be the ring of integers localized at 2 and, using the usual notation, put

$$
T=\left[\begin{array}{rr}
S & 2 S \\
S & S
\end{array}\right]
$$

Then $T$ is a semilocal hereditary Noetherian prime ring finitely generated over its centre. However, $T$ is not a maximal order. For if $I$ is the ideal

of $T$, and

$$
\left[\begin{array}{rr}
2 S & 2 S \\
S & S
\end{array}\right]
$$

$$
q=\left[\begin{array}{cc}
0 & 0 \\
1 / 2 & 0
\end{array}\right]
$$

then $q I \subset I$ and $q$ lies in the quotient ring of $T$, yet $q \notin T$.

We recall the definition of a maximal $C$-order from [5]. Let $C$ be a Krull domain with quotient field $K$, and $Q$ a finite dimensional central simple $K$-algebra. A $C$-order is, in the sense of Fossum, a subring $T$ of $Q$ satisfying:

(i) $C \subset T$;

(ii) $K . T=Q$;

(iii) $T$ is integral over $C$.

A $C$-order is called maximal if it is not properly contained in any $C$-order in $Q$. 
Suppose that $R$ is, in addition to our previous assumptions, a PI-ring. Proposition 1.5 of [2] now guarantees that $R$ is a maximal $Z$-order.

In particular we note that, by $[6,4.2 \mathrm{p} .147]$, for each $p \in \mathfrak{B}$ there is a unique prime ideal of $R$ lying over $p$. Each $R_{\mathrm{p}}$ is thus a local ring. Presumably this last statement remains valid without the additional PI hypothesis, but we have been unable to confirm this.

\section{REFERENCES}

1. K. A. Brown, C. R. Hajarnavis and A. B. MacEacharn, Rings of finite global dimension integral over their centres, Comm. Algebra, to appear.

2. M. Chamarie, Ordres maximaux et R-ordres maximaux, C.R. Acad. Sci. Paris Ser. A 285 (1977), 989-991.

3. M. Chamarie and A. Hudry, Anneaux Noetheriéns à droit entiers sur un sous-anneau de leur centre, Comm. Algebra 6 (1978), 203-222.

4. A. W. Chatters and C. R. Hajarnavis, Rings with chain conditions (Pitman, London 1980).

5. R. M. Fossum, Maximal orders over Krull domains, J. Algebra 10 (1968), 321-332.

6. G. Maury and J. Raynaud, Ordres maximaux au sens de K. Asano, Lecture Notes in Mathematics 808 (Springer-Verlag, 1980).

7. W. Vasconcelos, On quasi-local regular algebras, Symposia Mathematica 11 (1973), 11-22.

MATHEMATICS INSTTTUTE, UNIVERSITY OF WARWICK, COVENTRY CV4 7AL. 